\title{
LINE SHAPE EFFECTS ON INTENSITY MEASUREMENTS OF SOLAR FEATURES: BRIGHTNESS CORRECTION TO SOHO MDI CONTINUUM IMAGES
}

\author{
S. Criscuoli ${ }^{1}$, I. Ermolli ${ }^{1}$, D. Del Moro ${ }^{2}$, F. Giorgi $^{1}$, A. Tritschler ${ }^{3}$, H. Uitenbroek $^{3}$, And N. Vitas ${ }^{4}$ \\ ${ }^{1}$ INAF-Osservatorio Astronomico di Roma, Via Frascati 33, 00040 Monte Porzio Catone, Italy; serena.criscuoli@oaroma.inaf.it \\ ${ }^{2}$ Department of Physics, University of Roma Tor Vergata, Via della Ricerca Scientifica 1, I-00133 Roma, Italy \\ ${ }^{3}$ National Solar Observatory, Sacramento Peak, P.O. Box 62, Sunspot, NM 88349, USA \\ ${ }^{4}$ Sterrekundig Instituut, Utrecht University, Postbus 80 000, 3508 TA Utrecht, The Netherlands \\ Received 2010 November 5; accepted 2010 December 9; published 2011 January 25
}

\begin{abstract}
Continuum intensity observations obtained with the Michelson Doppler Imager (MDI) on board the SOHO mission provide long time series of filtergrams that are ideal for studying the evolution of large-scale phenomena in the solar atmosphere and their dependence on solar activity. These filtergrams, however, are not taken in a pure continuum spectral band, but are constructed from a proxy, namely a combination of filtergrams sampling the Ni I $6768 \AA$ line. We studied the sensitivity of this continuum proxy to the shape of the nickel line and to the degradation in the instrumental transmission profiles. We compared continuum intensity measurements near the nickel line with MDI proxy values in three sets of high-resolution spectro-polarimetric data obtained with the Interferometric Bidimensional Spectrometer, and in synthetic data, obtained from multi-dimensional simulations of magneto-convection and one-dimensional atmosphere models. We found that MDI continuum measurements require brightness corrections which depend on magnetic field strength, temperature and, to a smaller extent, plasma velocity. The correction ranges from $2 \%$ to $25 \%$ in sunspots, and is, on average, less than $2 \%$ for other features. The brightness correction also varies with position on the disk, with larger variations obtained for sunspots, and smaller variations obtained for quiet Sun, faculae, and micropores. Correction factors derived from observations agree with those deduced from the numerical simulations when observational effects are taken into account. Finally, we found that the investigated potential uncertainties in the transmission characteristics of MDI filters only slightly affect the brightness correction to proxy measurements.
\end{abstract}

Key words: Sun: photosphere - Sun: surface magnetism - techniques: image processing

Online-only material: color figures

\section{INTRODUCTION}

The continuum intensity measurements obtained with the Michelson Doppler Imager (MDI) have provided extensive time series of data unaffected by seeing for over 13 years. These data are ideal for studying large-scale phenomena in the solar atmosphere and their dependence on solar magnetic activity. They have been utilized in many investigations, concerning the analysis of large-scale patterns in plasma motions (e.g., Meunier et al. 2008, 2007, and references therein), the measurement of the radiative properties of magnetic elements over the activity cycle (e.g., Ortiz et al. 2002, 2006; Mathew et al. 2007), and the modeling of irradiance variations (e.g., Krivova et al. 2003; Wenzler et al. 2006, 2009).

Nevertheless, MDI continuum data are a by-product of the instrument, which was designed mainly for Doppler measurements. Specifically, intensities in the continuum are derived by the combination of five narrowband filtergrams, obtained with filters sampling a passband of $94 \mathrm{~m} \AA \mathrm{FWHM}$, equally spaced by $75 \mathrm{~m} \AA$ around the Ni I $6768 \AA$ mid-photospheric line. The filtergrams are labeled as $F_{0}, \ldots, F_{4}$, where $F_{0}$ is divided over two bands taken near the continuum, on either side of the line, $F_{1}$ and $F_{4}$ are centered on the wings of the nickel line, and $F_{2}$ and $F_{3}$ are centered around its core. Even at $F_{0}$ the instrument does not sample true continuum. Instead, a proxy continuumintensity filtergram is constructed by combining the five nominal filtergrams in the following way (Scherrer et al. 1995):

$$
I_{c}=2 F_{0}+I_{\text {depth }} / 2+I_{\text {ave }},
$$

where $I_{\text {ave }}$ is the average of the five filtergrams and $I_{\text {depth }}$ is the line depth, which is given by

$$
I_{\text {depth }}=\sqrt{2\left(\left(F_{1}-F_{3}\right)^{2}+\left(F_{2}-F_{4}\right)^{2}\right)} .
$$

The components of sum $I_{c}$ theoretically have canceling systematic errors as a function of solar velocity, so that the continuum image is claimed to be free of Doppler induced crosstalk at the $0.2 \%$ level (Scherrer et al. 1995).

The accuracy of the MDI continuum estimate (called the MDI method hereafter) is, however, inherently limited by the shape of the spectral line and its sensitivity to both strength and inclination of the magnetic field, local thermal stratification, and line-of-sight motions of the observed region. In addition, MDI measurements may also suffer from substantial errors because of uncertainties in the actual transmission characteristics of the filters utilized (Wachter 2008).

Several previous studies have focused on the accuracy of magnetic flux estimates obtained from MDI observations (e.g., Berger \& Lites 2003; Tran et al. 2005; Ulrich et al. 2009; Wang et al. 2009; Demidov \& Balthasar 2009; Zhendong et al. 2010), as well as the accuracy of dynamic measurements (Wachter et al. 2006; Rajaguru et al. 2007; Wachter 2008). Results indicate a substantial underestimate of magnetic flux and spurious contributions affecting Doppler measurements. This leads to recalibration of magnetograms and dopplergrams series, with the most recent results suggesting that the calibration of these data could be improved yet further. By contrast, the accuracy of continuum intensity measurements has only been investigated by Mathew et al. (2007), using spectral calculations based 
on one-dimensional atmospheric models. Their results indicate that MDI continuum measurements underestimate intensity in sunspots, with an error depending on both the magnetic field strength and temperature stratification.

The objective of this study is to further investigate the uncertainties affecting MDI continuum intensity measurements, by considering both high spatial resolution spectro-polarimetric observations and numerical simulations. To this aim we defined a brightness correction factor and investigated its variation with the physical properties of the analyzed solar features and observational conditions, such as spatial and spectral scattered light, finite spatial and spectral resolution, and position on the solar disk. We also investigated possible effects resulting from degradation of MDI filter transmission profiles.

The paper is organized as follows. We describe the data analyzed and provide some details of the reduction applied (Section 2). Then, we present the spectral synthesis method and the results obtained from our measurements and synthesis computations (Section 3). We also investigate the sensitivity of results to filter profiles uncertainties (Section 4). We finally discuss the results obtained and present our conclusions (Section 5).

\section{SPECTRAL DATA}

To investigate the sensitivity of the MDI continuum measurement proxy we employed profiles of the Ni I $6768 \AA$ line obtained from two different sources, namely high-resolution spectro-polarimetric observations acquired with the Interferometric Bidimensional Spectrometer (IBIS; Cavallini 2006; Reardon \& Cavallini 2008) at the NSO/Dunn Solar Telescope, as well as with theoretical line synthesis calculations, as described below.

\subsection{Observed Spectra}

The observed spectra used in this work were extracted from three IBIS data sets. These will be referred to as the Pore, Sunspot, and Faint Plage sets hereafter, alluding to the main solar features in their field-of-view (FOV). The Pore set is a spectral scan of the Ni I $6768 \AA$ line acquired near the disk center with diffraction limited spatial sampling at $0{ }^{\prime} 083$ pixel $^{-1}$. The Sunspot and the Faint Plage sets are fullStokes spectropolarimetric scan of the Ni I 6768 line, acquired at $\mu=0.88$ and $\mu=0.92$, respectively, with a spatial sampling of $0{ }^{\prime \prime} 17$ pixel $^{-1}$. The IBIS spectral profile at $6768 \AA$ has an FWHM of $25 \mathrm{~m} \AA$ (Reardon \& Cavallini 2008) and the spectral sampling was about $30 \mathrm{~m} \AA$ for the three sets. The data sets were reduced following standard procedures (Cauzzi et al. 2008; Viticchié et al. 2009; Judge et al. 2010) which also correct for the instrumental blueshift, and for instrument and telescope polarization in the case of spectropolarimetric data. The pipeline includes an MFBD image reconstruction technique (van Noort et al. 2005) to reduce seeing degradation and possible seeing induced crosstalk.

Note that all observed line profiles suffer from finite spectral resolution and spectral scattered light contamination. This last effect was estimated by comparison of observed average quiet-Sun profile with Atlas measurements by Kurucz et al. (1984), following the procedure described in Cabrera Solana et al. (2007, their Equation (1)). We found a consistent light contamination level of $12 \%$ on all the three data sets analyzed.

To estimate the magnetic flux density along the line-of-sight $\left(B_{\mathrm{LOS}}\right)$ in image pixels of the Sunspot and Faint Plage sets,

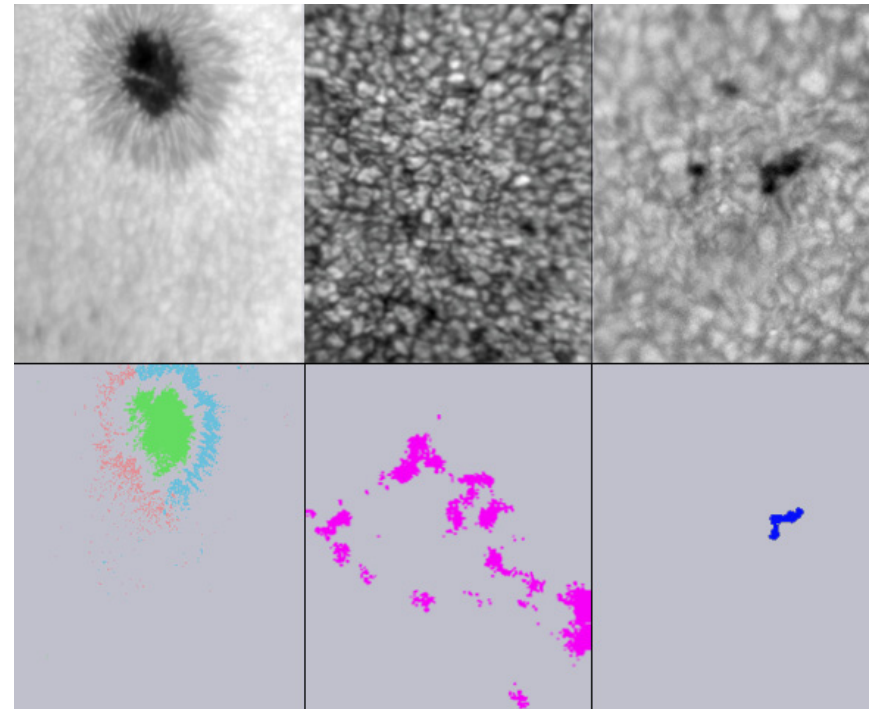

Figure 1. Top: subfields of 40 arcsec $\times 50$ arcsec of frames from Sunspot (left), Faint Plage (middle), and Pore (right) data sets. Bottom: masks depicting features identified according to criteria in Table 1. The color code is the same as in Figure 2.

(A color version of this figure is available in the online journal.)

we applied the center of gravity method (COG) by Rees \& Semel (1979). This method provides a reasonable estimate of the $B_{\mathrm{LOS}}$ at the formation height of the line core as demonstrated by Uitenbroek (2003) for several lines of Fe I. We applied the method to synthetic sunspot profiles from the three-dimensional MHD simulations (described in Section 2.2) taking into account both spatial and spectral scattered light and found that the $\mathrm{COG}$ in the Ni I line can underestimate magnetic flux by up to $\approx 28 \%$ for $B_{\mathrm{LOS}}>1.5 \mathrm{kG}$. The $B_{\mathrm{LOS}}$ value deduced for each image pixel was compensated for LOS projection by applying the relation (Landi \& Landolfi 2004, p. 660)

$$
B=B_{\mathrm{LOS}} / \cos \gamma
$$

where the inclination angle $\gamma$ derives from

$$
\gamma=\arccos \frac{\sqrt{1+x^{2}}-1}{x}
$$

$x=V / \sqrt{U^{2}+Q^{2}}$, and $x$ is computed at the left wing of the line.

As an aid in classification of the observed pixels (see Table 1), we computed the total polarization signal $T$ in each image pixel, which is defined as

$$
\mathcal{T}=\int\left(Q^{2}+U^{2}+V^{2}\right)^{1 / 2} / I_{c} d \lambda,
$$

where $I_{c}$ is the intensity in the continuum near the Ni I line.

Finally, line-of-sight velocities were estimated by Doppler shifts of line cores with respect to the average quiet-Sun line center position.

Characteristic maps of the continuum intensity $I_{C}$ of each set are shown in Figure 1. The FOVs include a large variety of solar features, characterized by different physical properties and therefore different shapes of line profiles. An example of such variety is reported in Figure 2, which shows average line profiles of pixels selected according to the criteria described in Table 1. 


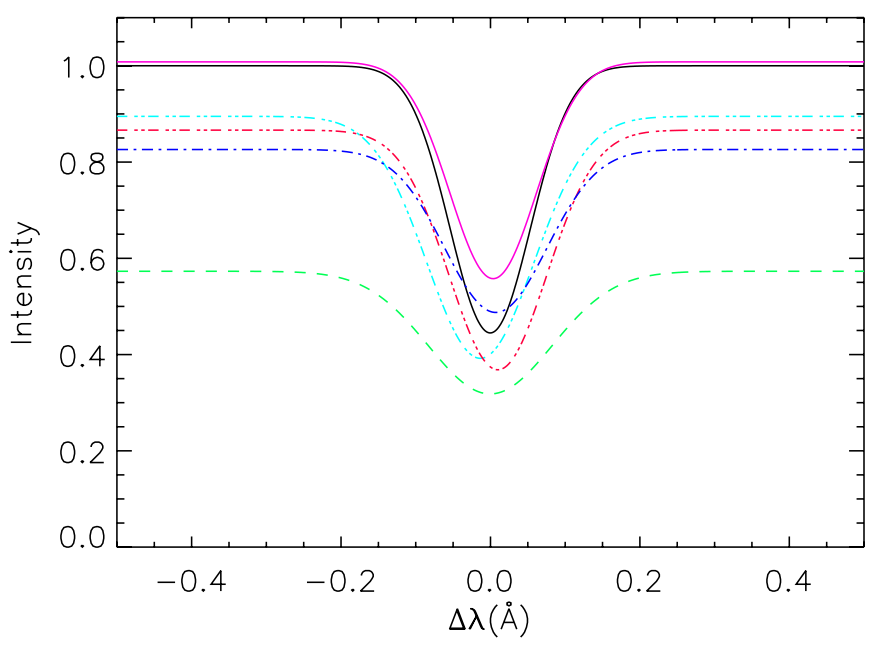

Figure 2. Line profiles, computed by Gaussian fits to average spectral intensities of various features singled out on the IBIS observations as indicated in Table 1. Continuous black: quiet Sun. Continuous magenta: facula. Dashed green: umbra. Dot-dashed blue: pore. Triple-dot-dashed light blue: blueshifted penumbra. Triple-dot-dashed red: redshifted penumbra.

(A color version of this figure is available in the online journal.)

Table 1

Criteria Utilized for the Identification of the Solar Features Depicted by Masks in Figure 1 and Whose Line Profiles are Shown in Figure 2

\begin{tabular}{lcc}
\hline \hline \multicolumn{1}{c}{ Solar Feature } & Criterion & Data Set \\
\hline Quiet Sun & $T \leqslant 3.0 \mathrm{m \AA}$ & $\begin{array}{c}\text { Spot } \\
\text { Faint Plage } \\
\text { Facula }\end{array}$ \\
& $B_{\mathrm{LOS}} \geqslant 0.2 \mathrm{kG}$ & Faint Plage \\
and $C_{\text {core }} \geqslant 10 \%$ & Spot \\
Umbra & $B_{\mathrm{LOS}} \geqslant 1.5 \mathrm{kG}$ & Spot \\
Penumbra blueshift & $0.8 \mathrm{kG} \leqslant B_{\mathrm{LOS}} \leqslant 1.1 \mathrm{kG}$ & Spot \\
Penumbra redshift & and $v_{\mathrm{LOS}} \leqslant 0$ & \\
& $0.8 \mathrm{kG} \leqslant B_{\mathrm{LOS}} \leqslant 1.1 \mathrm{kG}$ & Pore \\
\hline
\end{tabular}

Notes. $B_{\mathrm{LOS}}$ indicates the magnetic flux strength along the line of sight, $C_{\text {core }}$ is the intensity contrast in the $\mathrm{Ni}$ I line core that is defined by the ratio $I / I_{\mathrm{QS}}$, where $I_{\mathrm{QS}}$ is the quiet-Sun intensity, $v_{\mathrm{LOS}}$ indicate the velocity of plasma along the LOS, and ave and $\sigma$ are the average and standard deviation of all the intensity values measured on the FOV.

\subsection{Synthetic Spectra}

To better understand the results obtained from observations and to distinguish between observational and methodological effects, we also computed synthetic spectra of the Ni I line by utilizing various numerical atmospheres: two two-dimensional cross sections from a three-dimensional simulation of magnetoconvection of an active region with average vertical field of $250 \mathrm{G}$ (Stein \& Nordlund 1998), a vertical cross section of an umbral magneto-convection simulation (Vitas et al. 2010), and various one-dimensional hydrostatic models. The onedimensional static models we considered ranged from the semiempirical umbral models E, L, and M from Maltby et al. (1986), the model by Socas-Navarro et al. (2004, Socas, hereafter) to the most recent radiative equilibrium models of Kurucz. ${ }^{5}$ In all the Maltby and Kurucz models, we assumed vertical height-independent magnetic fields. For the Maltby models, we assumed magnetic field strengths ranging from $2.0 \mathrm{kG}$ to $4.0 \mathrm{kG}$. The full set of one-dimensional models, which are

\footnotetext{
5 http://wwwuser.oat.ts.astro.it/castelli/grids.html
}

Table 2

One-dimensional Atmosphere Models Considered

\begin{tabular}{lc}
\hline \hline Model & $B(\mathrm{kG})$ \\
\hline Kur_5.50 & 1.10 \\
Kur_5.25 & 1.70 \\
Kur_5.00 & 2.25 \\
Kur_4.75 & 2.25 \\
Kur_4.50 & 2.50 \\
Kur_4.25 & 3.00 \\
Kur_4.00 & 3.50 \\
Kur_3.75 & 4.00 \\
Maltby L & 2.00 \\
Maltby L & 2.50 \\
Maltby L & 3.00 \\
Maltby M & 2.50 \\
Maltby M & 3.00 \\
Maltby M & 3.50 \\
Maltby E & 3.00 \\
Maltby E & 3.50 \\
Maltby E & 4.00 \\
Socas & 2.3 \\
\hline
\end{tabular}

Notes. Kurucz models are indicated with Kur_XXX where $\mathrm{XXX}$ is the effective temperature in $10^{3} \mathrm{~K}$ units.

all representative of umbral regions, and the corresponding magnetic field strengths are listed in Table 2. The value listed for the Socas model in this table, however, is the magnetic field strength of $\tau_{5000}=1$, since this model was used with its original depth-dependent magnetic field structure. Note that we set the turbulent velocity value in Kurucz models to zero, since other values in these models are more representative of non-magnetic and convectively unstable atmospheres.

The spectral synthesis was performed in non-local thermal equilibrium with the RH code by Uitenbroek (2002, 2003), and employing the model atom of Bruls (1993). The emergent spectra were calculated at various lines of sight for a $1 \AA$ wide spectral interval centered on the Ni I line. Figure 3 shows examples of line profiles derived from the synthesis in the various atmospheres, all normalized to the intensity value calculated from the FAL-C model of Fontenla et al. (1993), which is representative of the quiet Sun. This normalization provides a common intensity reference value for the different simulations. Similarly to observations, the line profiles derived from the synthesis display a large variety of shapes, because of the different physical conditions in the simulated atmospheres. The synthetic profiles differ from those deduced from observations of magnetic features mostly in terms of the line core reversal. This must be mainly ascribed to the transverse Zeeman component (Stix 2004), which, especially for high values of magnetic field strength, can considerably modify the shape of the line core, and the fact that all observations were taken slightly off disk center.

\section{SIMULATION OF MDI CONTINUUM MEASUREMENTS}

To test the algorithm for the MDI continuum proxy we compare the values it predicts with the real continuum values in the observed and simulated spectra of the Ni I 6768 line. We first evaluate the intensities in each of the five filter positions by multiplying the spectra with the filter passband and integrating over wavelength, then add the integrated intensities according to Equation (1). The MDI transmission profiles were centered 

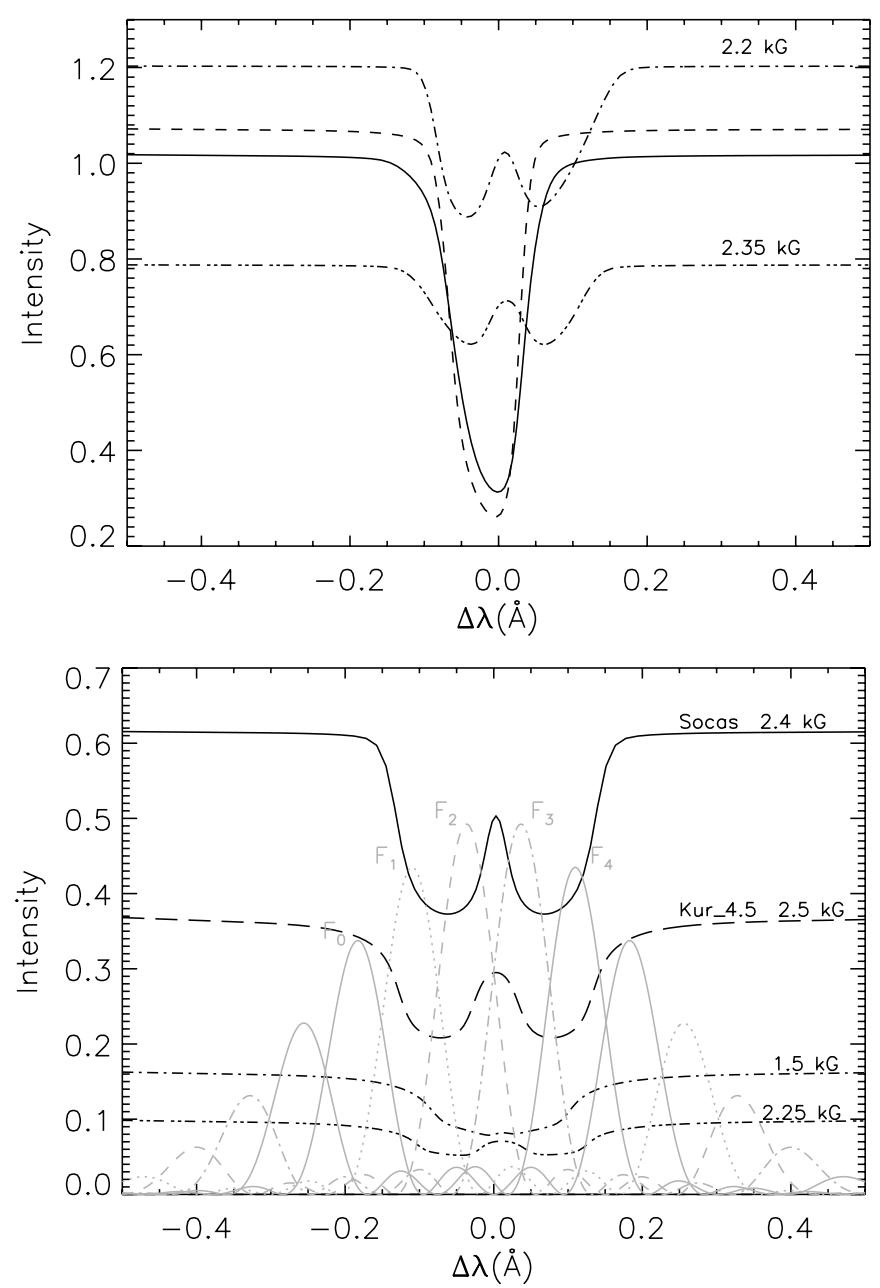

Figure 3. Line profiles derived from the numerical simulations. Top panel: results from the active region magneto-convection simulation. Solid: quiet Sun. Dashed: upflow. Dot-dashed: bright magnetic feature (facula). Dot-dot-dashed: dark magnetic feature (micropore). Bottom panel: results from hydrostatic models and magneto-convection simulations of sunspot. Overlaid profiles show MDI spectral transmission profiles normalized to fit the figure.

on the core of the average line profile of the quiet Sun. This was defined as in Table 1 in the case of Sunspot and Faint Plage data set, while for the Pore data set we considered an area in the FOV of $80 \operatorname{arcsec}^{2}$ far from the pores. As for simulations, we centered the transmission profiles to the core of the line profile derived from the FAL-C model.

Since we generally do not measure absolute intensities in observations, but rather intensities relative to some average intensity, we analyze validity of the MDI continuum estimate by measuring contrast, where contrast is defined as the ratio between the intensity of an analyzed feature and the intensity of the quiet Sun. For observed spectra, the contrast was estimated after compensation for the center-to-limb variation (CLV) of quiet-Sun intensity across the FOV on each frame. The CLV was derived from the second-order polynomial surface best fitting the intensity pattern of quiet-Sun regions in the image, after masking dark magnetic features by applying an intensity criterion thresholding, and application of a smoothing function to pixel intensities. Concerning numerical simulations, intensity values were normalized by the intensity at the $\mathrm{Ni}$ I continuum calculated with the FAL-C model for the appropriate disk positions. Furthermore, we define as brightness correction factor to MDI continua measurements the ratio $C / C_{\mathrm{MDI}}$, where $C$

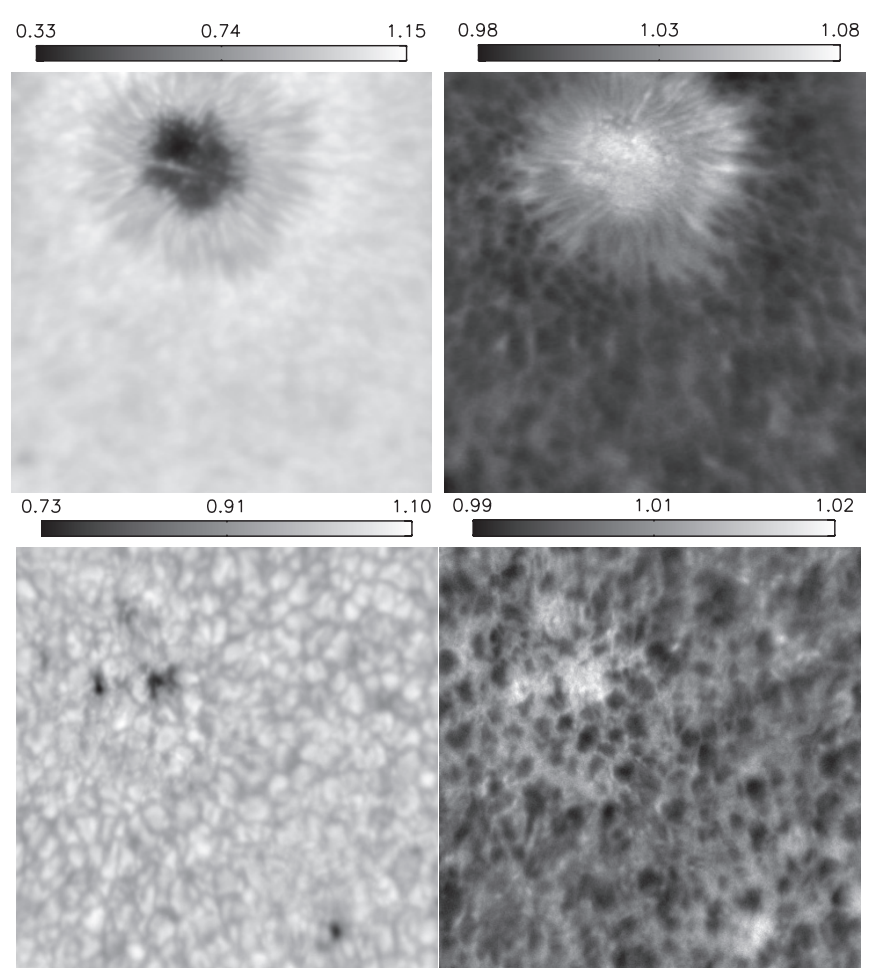

Figure 4. Left panels: continuum contrast maps derived from Sunspot (top) and Pore (bottom) data sets. Right panels: corresponding maps of the brightness correction.

is the contrast value obtained in the continuum near the $\mathrm{Ni}$ I line (hereafter called the continuum contrast) and $C_{\mathrm{MDI}}$ is the contrast derived with the MDI method (hereafter called the MDI contrast). In the following, we investigate the dependence of this correction factor on various properties of the analyzed regions, e.g., the contrast, magnetic field strength, and plasma velocity, as well as observational conditions.

\subsection{Results from Observed Spectra}

Figure 4 shows maps of the intensity contrast (left panels) in the Sunspot (top) and Pore (bottom) sets and the corresponding maps of the brightness correction to MDI measurements (right panels). We found that the correction factor is, on average, lower than $2 \%$ of the MDI contrast, but increases up to $8 \%-10 \%$ in regions with high magnetic field strength. The figure also shows that the MDI contrast is, on average, overestimated in upflow regions (granules), while it is underestimated in regions with downflows (intergranular lanes), and in particular in regions with strong magnetic fields. The map of the brightness correction in the Pore (lower right panel) looks very similar to a map of the inverse granulation as it would appear in core intensity of a photospheric spectral line, implying that the correction factor depends strongly on the photospheric temperature gradient, which is steep over granules and shallow over intergranular lanes. The reason that the MDI continuum estimate depends on this temperature gradient lies in the corresponding widths of the spectral line profile, which are narrow and deep in granules, and wide and shallow over intergranular lanes. Profiles that are wider than that of the average quiet Sun cause an underestimate of the line depth (Equation (2)), and consequentially an underestimate of the MDI continuum (Equation (1)). The effect is also visually enhanced by the fact that magnetic field concentrations preferentially reside in downflow regions. This is further explained in Section 3.2. 


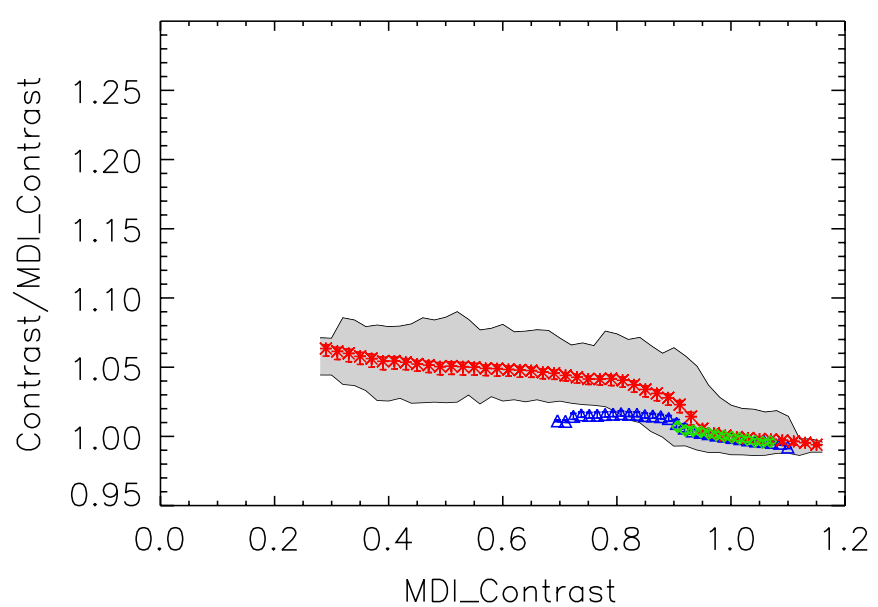

Figure 5. Dependence of the brightness correction factor on the continuum contrast derived with MDI method. Red asterisks, blue triangles, and green diamonds show values derived from averages over contrast bins, each 0.02 wide, on the Sunspot, Pore, and Faint Plage sets, respectively. The error bars represent the standard deviations of the values in each bin. Shaded area delimits the values within $3 \%$ of the maximum of the distribution of values in each bin for the Sunspot data set.

(A color version of this figure is available in the online journal.)

Figure 5 shows the brightness correction to the MDI contrasts derived from all the image pixels in all three data sets. Symbols and error bars of a given color show the average and standard deviation of the correction factor derived from a given data set by considering the MDI contrast values for the set collected into bins with 0.02 width. We found that the brightness correction is close to unity for bright features, because of the opposite contribution from Doppler shifts and magnetic fields to the line shape, though we note some dispersion of results. On the other hand, the correction factor increases with the decrease of the MDI contrast derived from the images, reaching 1.08 for MDI contrasts $\leqslant 0.3$.

Next, we investigated the effects of the magnetic field strength on MDI contrast estimates in more detail. Figure 6 shows the results derived from a sample of Sunspot and Faint Plage images (seven and five IBIS line scans, respectively). We found that the brightness correction increases with the increase of the magnetic field strength, although a saturation value of $\approx 1.04$ is reached at magnetic flux larger than $1.5 \mathrm{kG}$. In spite of the small standard deviation values, we also note a large scatter of results, especially for the regions with $|\Phi(B)| \leqslant 1.5 \mathrm{kG}$. In the range $0.7 \mathrm{kG} \leqslant|\Phi(B)| \leqslant 1.2 \mathrm{kG}$ this is due to the combined effect of LOS velocity and magnetic field strength, since this range also comprises pixels belonging to the sunspot penumbra. At lower values of magnetic flux the large scatter of results likely derives from uncertainties in the COG estimations of the magnetic field, which are due to limited spatial resolution and residual cross-talk in Stokes signals.

Finally, we investigated the effects of LOS velocity on brightness correction, restricting the analysis to finite ranges of magnetic field strength values. Results obtained for quiet-Sun regions selected on the three data sets are shown in Figure 7. In agreement with visual inspection of images in Figure 4 the brightness correction is, on average, larger than unity for downflow regions and smaller than unity for upflow regions, but with large dispersion. Due to the lower image quality of Pore and Faint Plage data sets, this effect is more evident on the Sunspot data.

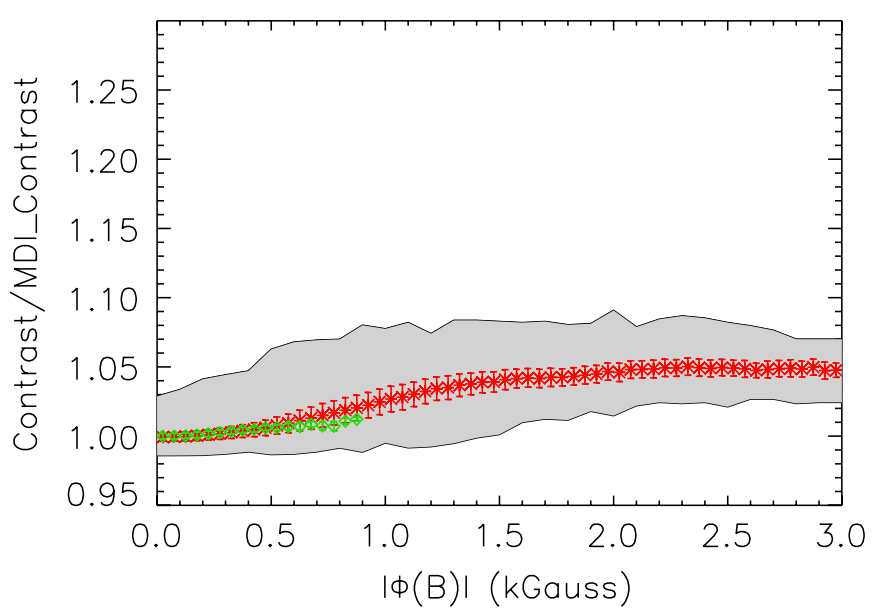

Figure 6. Dependence of the brightness correction on the magnetic flux of the analyzed region. Legend as in Figure 5.

(A color version of this figure is available in the online journal.)

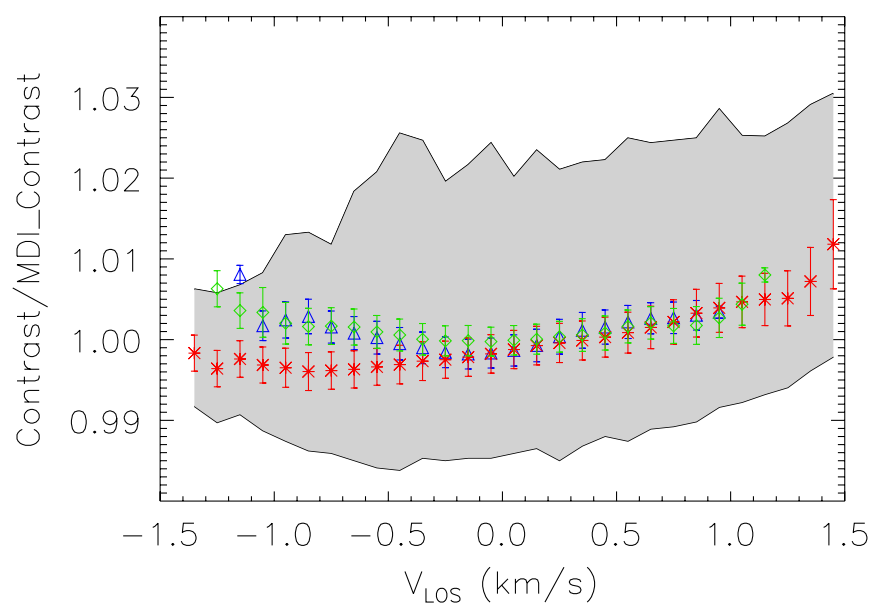

Figure 7. Dependence of the brightness correction on the LOS velocity for quietSun regions identified on the three IBIS data set. Negative velocities correspond to upflow motions, positive to downflow motions. Legend as in Figure 5.

(A color version of this figure is available in the online journal.)

\subsection{Results from Synthetic Spectra}

Our analysis of observations performed with the observed Ni I spectra indicates that the brightness correction to MDI measurements depends on the solar feature analyzed. This is confirmed by the results derived from various numerical simulations. Similarly to Figure 5 derived from observations, Figure 8 shows the brightness correction deduced from the various atmospheres described in Section 2. Here the set of points with contrast close to unity stems from the magneto-convection simulation of the active region, the set of points with contrast close to 0.1 stems from the magneto-convection simulation of sunspot umbra, and the intermediate contrast points stem from hydrostatic models. We found that the brightness correction is close to unity, with some dispersion of results due to magnetic field and velocity effects, for MDI contrast values ranging from 0.8 to 1.2 , as also derived from the three IBIS data sets. However, the correction factor increases up to $1.15-1.25$ for MDI contrast $\leqslant 0.5$, i.e., by a larger fraction than derived from observations. We also found a large dispersion of results from the magneto-convection simulations of umbral regions, because of the wide range of field strengths in the analyzed atmosphere models. The dispersion of correction values from simulations is also larger than those from observations. 


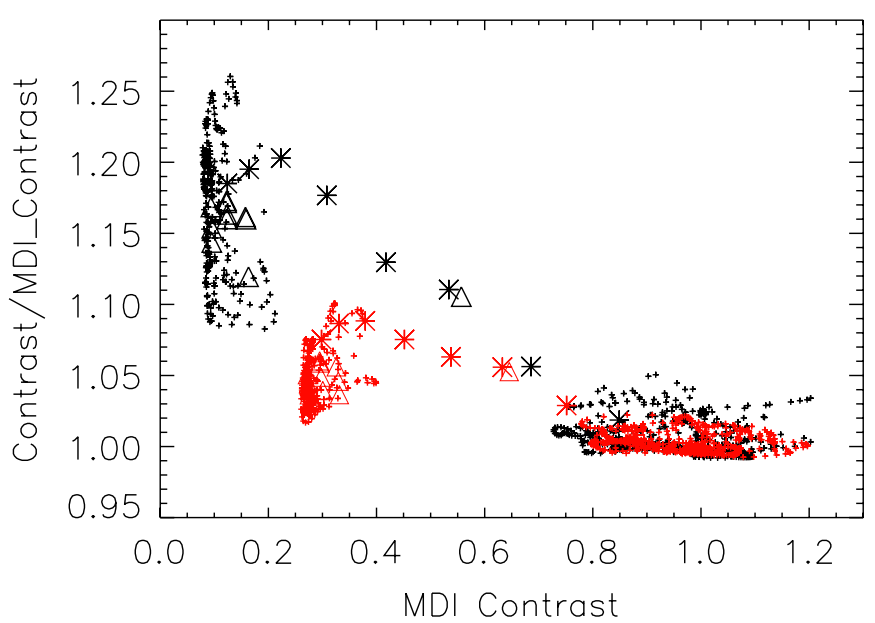

Figure 8. Dependence of the brightness correction to the MDI contrast computed for the various atmospheres considered in this study. Crosses: results from the magneto-convection calculations. Asterisks: Kurucz models. Triangles: Maltby and Socas models. Black: results obtained at disk center. Red: results obtained at disk position $\mu=0.88$ after data degradation to account for the finite spectral resolution, spurious light and scattered light degradations affecting the IBIS data

(A color version of this figure is available in the online journal.)

To assess the importance of observational effects on the results derived from the three data sets and to place them in context with the results deduced from model calculations, we computed synthetic spectra from the various model atmospheres at the heliocentric angles of our observations (e.g., $\mu=0.88$ for the Sunspot set) and degraded the results to account for the finite spectral resolution, spurious spectral light contamination, and spatially scattered light degradation affecting the observations.

This latter effect was mimicked by applying the formula $I_{S}=(1-\alpha) I+\alpha I_{q}$, where $\alpha$ indicates the level of spurious spatial light contamination, and $I_{q}$ is the quiet-Sun intensity value. Estimation and compensation on high spatial resolution data for scattered light results incomplete, even employing the most sophisticated restoration algorithm available (e.g., MOMFBD; Scharmer et al. 2010). In the lack of measurements, we assumed the value $\alpha=0.2$, which is within the range of values $(0.17-0.25)$ found by Briand et al. (2006) for IPM/THEMIS data. The results obtained indicate that the observational conditions and degradations above listed all affect the brightness correction, specifically by decreasing its value. The difference between the three IBIS data sets must therefore be ascribed to the different image qualities. We also note that, among the observational effects considered, the brightness correction is affected the most by spurious spatial light contamination, which also contributes to a smaller dispersion of the results from observations (Mathew et al. 2007; Criscuoli \& Ermolli 2008).

Figure 9 shows the dependence of the brightness correction on the magnetic field strength in the synthetic atmospheres. The results deduced with simulations are qualitatively in agreement with those derived from observations, though the saturation value is reached only at $B=3 \mathrm{kG}$ and the dispersion of results is larger than obtained from observations as a result of image degradation by limited spatial resolution and spatially spurious light. The plot clearly shows a double distribution: one for which the brightness correction value is smaller than 1.05 and one for which it is higher. We found that data from the active region magneto-convection simulation belong to the first group, whereas the second group is populated by sunspot models (with

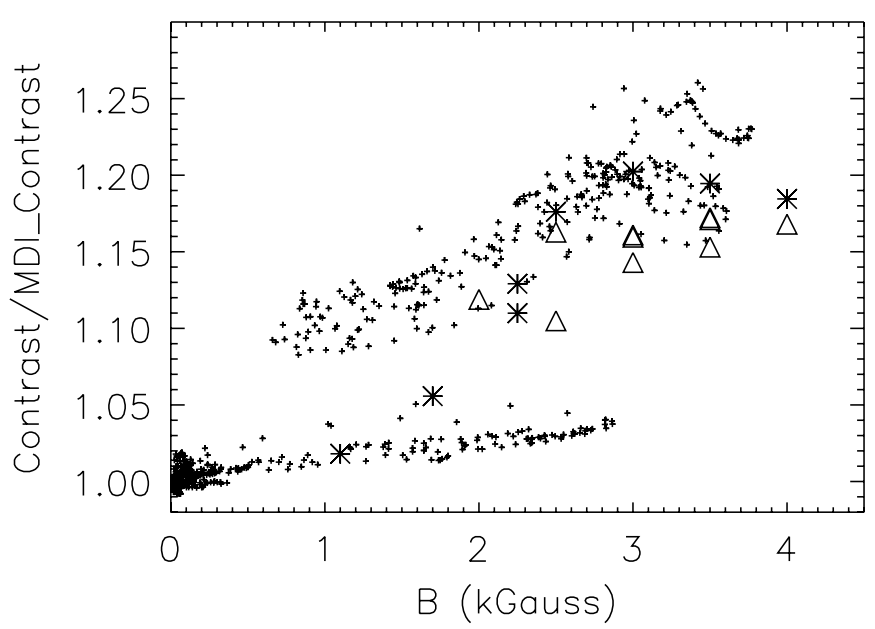

Figure 9. Dependence of the brightness correction on the magnetic field strength derived from synthetic spectra. Legend as in Figure 8.

the exception of the two hottest Kurucz models) and the umbral simulation.

We found that the MDI continuum estimate method depends in a complicated way on both temperature and magnetic field intensity. The larger the magnetic field strength, the wider the line profile. If the line profile becomes so wide and/or shifted that it reaches into (one or two of) the outermost transmission $F_{0}$ passbands, the continuum (Equation (1)) is underestimated, resulting in a brightness correction larger than one. This effect is also discussed in Mathew et al. (2007) and illustrated in their Figure 7. These authors also note that for the largest magnetic field values considered in their study, a saturation of the error occurs because of the filling in of the line core, as is also clear in the line profiles in Figure 3. By contrast, an increase in the effective temperature narrows the line and increases its depth (Mathew et al. 2007, their Figure 8). As a consequence, for a given value of magnetic field strength the brightness correction is smaller for faculae rather than for sunspots. On the other hand, at the saturation limit, where the field mostly affects the width of the line, the error in the MDI continuum estimate increases with the effective temperature as the line narrows. We also note that the brightness correction factor for the synthesized spectra (Figure 9) seems less affected by saturation than the one for the observed spectra (Figure 6).

Next, we investigated the dependence of the brightness correction on the LOS velocity of the plasma. We found that, on average, the brightness correction is larger than unity and increases with the magnitude of the LOS velocity. This is illustrated in Figure 10, which shows the results derived for quiet-Sun portions (defined here by those pixels with $B \leqslant$ $50 \mathrm{G}$ ) of the magneto-convection simulations representative of active region. As we can see, data from simulations allow us to investigate a wider range of velocities with respect to observations (Cauzzi et al. 2006). When restricting the analyses to the range from $-2 \mathrm{~km} \mathrm{~s}^{-1}$ to $2 \mathrm{~km} \mathrm{~s}^{-1}$ we note the same asymmetry we found for observations, namely a brightness correction smaller than unity for upflows and larger than unity for downflows. This asymmetry is the result of the correlation between temperature gradient and up- or downflows in the granulation. Granules correspond to upflows (negative $v_{\text {LOS }}$ ) and have steep temperature gradients resulting in narrow and deep profiles. The shallow temperature gradient over intergranular lanes, where most downflows occur, leads to broader profiles. 


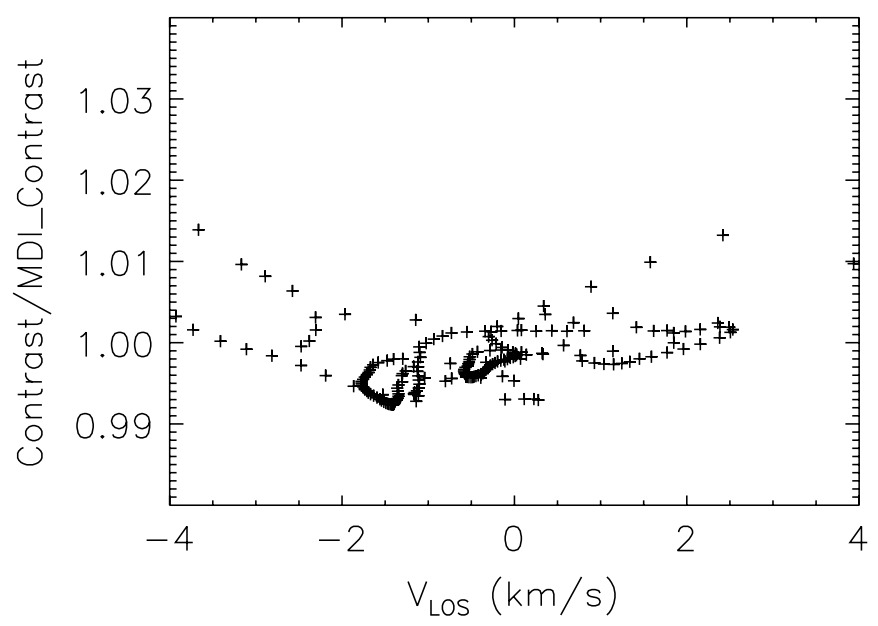

Figure 10. Dependence of the brightness correction to MDI measurements on the LOS velocity for quiet regions from magneto-convection simulation of the active region.

The spacing of the MDI filter positions is chosen such that it matches the width of the average quiet-Sun profile of the nickel line in the sense that Equation (2) gives the proper line depth. For substantially narrower profiles the filter positions $F_{1}$ and $F_{4}$ sample close to continuum values increasing the line depth estimate, overestimating the continuum. This leads to the correction factors below unity for small upflows. For larger line shifts to either sides, but already for smaller velocities in the downflows with their wider profiles, the correction factor is larger than one, because $F_{1}$ and $F_{2}$ (for blueshifts), or $F_{3}$ and $F_{4}$ (for red shifts) both end up close to the core, minimizing their difference in sampled intensity, underestimating the line depth, and underestimating the continuum level.

Finally, we considered the dependence of the brightness correction on the position of the analyzed feature on the solar disk. To this aim, we computed the synthetic spectra for 13 different viewing angles from various model atmospheres. In particular, we employed all the static sunspot models as well as the magneto-convection simulations representative of an active region. The nickel line forms low enough in these models so that the vertical domain includes its formation height, even at high inclinations of the LOS. Figure 11 shows the correction factor for the different Kurucz models with cooler models and stronger fields (both leading to wider profiles and larger correction factors) toward the top. For the models with $B \leqslant 3 \mathrm{kG}$ (or $T_{\text {eff }} \geqslant$ $4500 \mathrm{~K}$ ), the brightness correction monotonically decreases with the decrease of the heliocentric angle, because the width of the line decreases with the line-of-sight component of the magnetic field, which changes linearly with $\mu$ for the assumed vertical fields. For models with $B>3 \mathrm{kG}\left(T_{\text {eff }} \leqslant 4250 \mathrm{~K}\right)$, the CLV of the correction factor increases strongly with decreasing $\mu$ from 1.0 to 0.9 , and then decreases again monotonically with decreasing $\mu$. The initial rise is the result of the transverse magnetic Zeeman effect, which produces intensity decrease of the core of the profile for positions close to disk center, thus creating characteristic double-peaked profiles. These quickly disappear for $\mu<0.9$. Figure 11 also shows the CLV of the correction factor for faculae (squares), designated as those areas that have a field strength larger than $0.2 \mathrm{kG}$ and contrast greater than one) in the magneto-convection active region simulation. These facular regions show very little variation in their required correction factors across the disk.

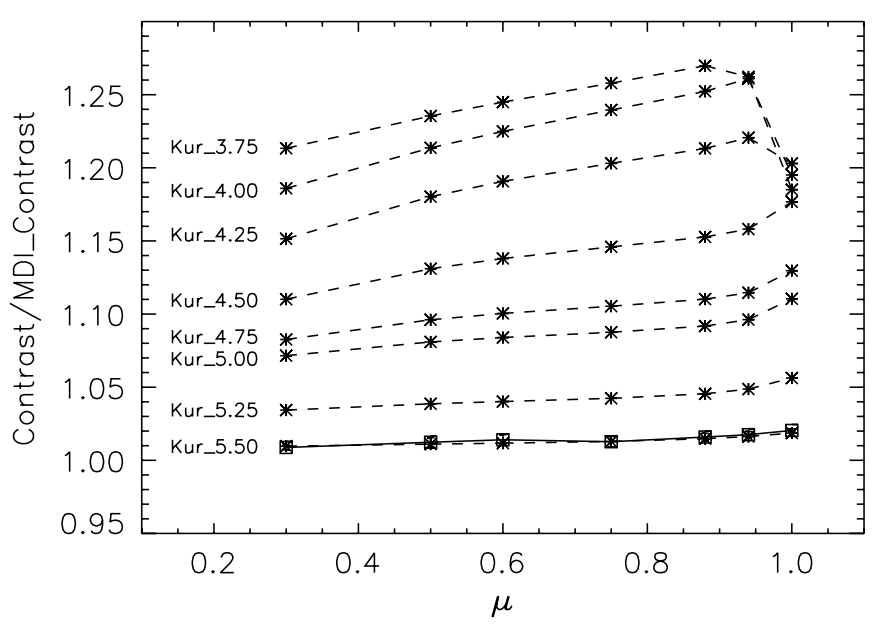

Figure 11. Center to Limb variation of the brightness correction for various magnetic features investigated through synthetic spectra. Squares: faculae, from magneto-convection simulation. Asterisks: sunspots, from Kurucz models.

\section{SENSITIVITY TO PASSBAND UNCERTAINTIES}

To assess the importance of an inaccurate knowledge of the filter characteristics, we analyzed the dependence of the brightness correction derived from observed spectra on potential inaccuracies in the precise tuning and width of the MDI passbands. In particular, we computed the variation of the brightness correction for displacements of the $F_{0}, \ldots, F_{4}$ passband pattern ranging from $-0.14 \AA$ to $+0.14 \AA$ from its nominal position. These values correspond to Doppler shifts of $\pm 6 \mathrm{~km} \mathrm{~s}^{-1}$ and are 1.5 times larger than the ones considered in the calibration of the MDI measurements (Scherrer et al. 1995, their Figure 12). We also investigated the sensitivity of the required brightness correction to variations of the width of the MDI passband respect to the nominal value of $94 \mathrm{~m} \AA$.

Figure 12 displays the resulting brightness correction as a function of displacement of the MDI passband pattern, when considering the same average observed line profiles for quiet Sun, plage, pore, umbra, and the most blueshifted penumbra (see Figure 2). We found that, for moderate displacement values, the brightness correction decreases for displacements in the same direction as the line profile Doppler shift, while it increases for shifts in the opposite direction. This is particularly clear for the penumbral profile (triple-dot-dashed curve). For large displacements all the curves in Figure 12 show a trend toward unity, because the offset between the line center and transmission pattern becomes so large that the passbands sample more and more true continuum. Note also the oscillatory variations of the curves, that, as pointed out by Wachter (2008), have a period proportional to the spacing of the transmission profiles of the filters.

Next, we computed variation of the brightness correction for different assumed widths of the tunable MDI passband by convolving the nominal profiles with Gaussians of widths that are multiples of the width of the profiles. This last quantity was estimated fitting the highest lobe of $F_{0}$ with a Gaussian function. Figure 12 shows the results for the various solar features. We found that the brightness correction decreases with the increase of the filter profile widths, and eventually drops below unity for the pore and facular profiles. This is the result of the transmission profile becoming wider than the line profile (see also the discussion in Section 3.2). Note also that the brightness correction for the penumbra is the least affected by the widening 

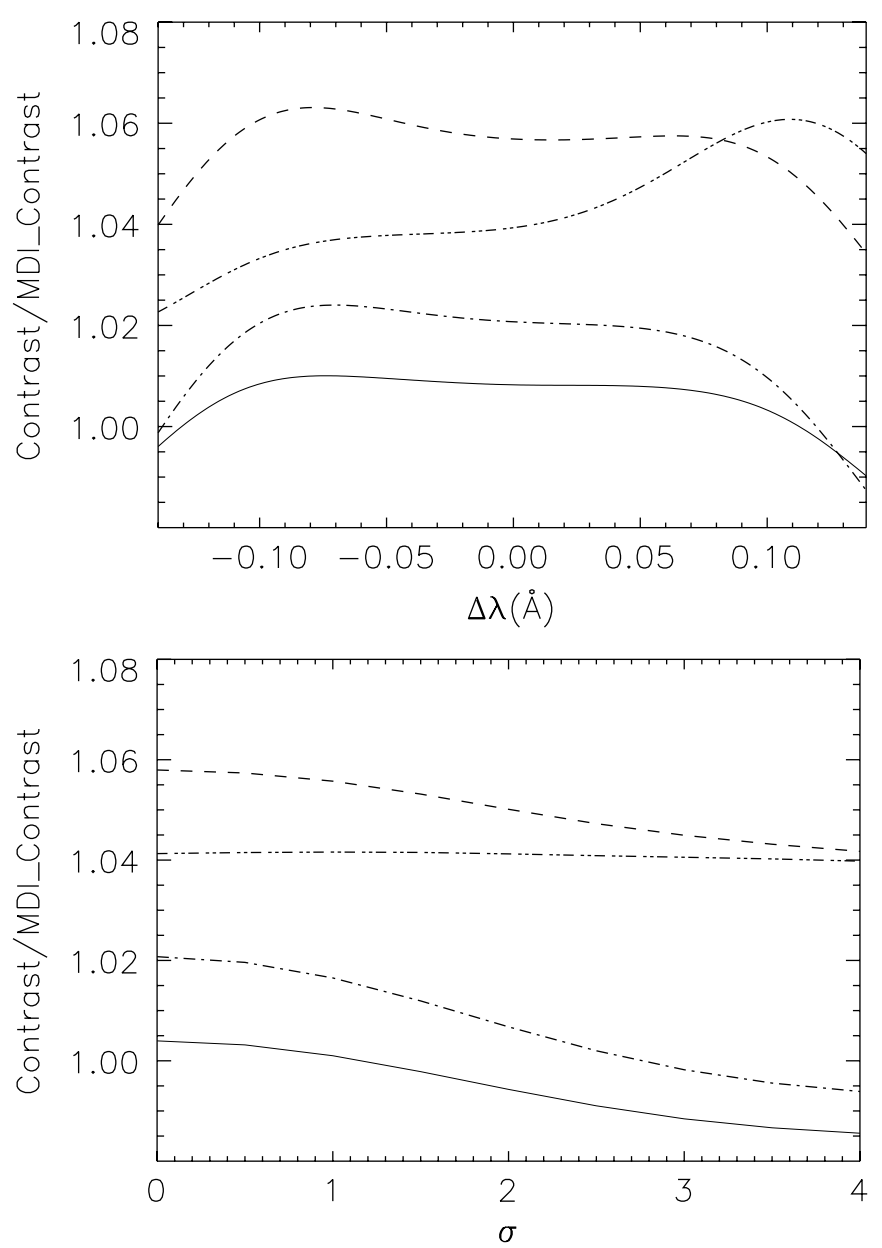

Figure 12. Variation of brightness correction of features identified on IBIS data (see Table 1) with the filter drifts (top panel) and modifications of shapes of transmission profiles (bottom panel) considered in this study. Continuous line: plage region. Dashed line: umbra. Dot-dashed line: pore. Triple-dot-dashed line: penumbra.

of transmission line profiles, since its value is mostly determined by the shift of the spectral line.

\section{DISCUSSION AND CONCLUSIONS}

We have presented a study of the accuracy of the MDI continuum intensity measurement, with the help of high spatial resolution spectro-polarimetric observations acquired with the IBIS/ DST and outcomes from numerical simulations representative of different solar regions. In order to provide the reader with a quantity that clearly indicates the MDI uncertainties and allows their compensation on measurements, we defined as brightness correction the ratio between the contrast value in the continuum and the one estimated through the MDI method and investigated the variation of this quantity with the physical properties of analyzed solar regions.

We found that the MDI brightness correction, on average, increases with the increase of the LOS magnetic field strength, the decrease of temperature, and the increase of LOS velocity. To summarize, the contrast is overestimated in quiet upflow regions, and is underestimated in magnetic and quiet downflow regions. The amount of correction is less than $1 \%$ in quiet regions and increases with the magnetic field strengths, reaching values up to $25 \%$ of the MDI continuum value in sunspots. However, line saturation effects limit the MDI error in regions with the highest magnetic field strengths.
Results derived from observations agree with those deduced from simulations, when accounting for the observational conditions and instrumental degradations affecting the observations. Among the effects considered, spatially spurious light is the one that most affects the brightness correction values. In the lack of a reliable estimate on our observational data, we therefore refrained from a direct comparison of results from MDI and IBIS data. On the other hand, we did not find any significant variation of the brightness correction values when degrading the results from IBIS data to the spatial resolution of MDI, thus suggesting that brightness correction is not significantly dependent on the spatial resolution of the observations.

Note that the brightness correction values that we found correspond to errors much higher than the $0.2 \%$ quoted in the instrument description of Scherrer et al. (1995, p. 163).

The brightness correction factor derived from synthetic spectra also depends on the position on the solar disk in different manners for the different features analyzed. We found that the brightness correction for sunspot models with strong field $\left(B_{\mathrm{LOS}} \geqslant 3 \mathrm{kG}\right)$ varies by up $2 \%$ for disk positions corresponding to $0.9 \leqslant \mu \leqslant 1$, reaching a maximum value for $\mu=0.9$, and then decreasing toward the limb. For weaker fields the variation across the disk is much less (Figure 11). Also for quiet Sun, faculae, and micropores the variation in brightness correction is small, below $1 \%$, across the disk.

Finally, we investigated effects of possible filter degradations on MDI continuum contrast estimates. We estimated variations with the shift of the passband pattern and with the change in the width of the transmission profiles of the instrument. Results are summarized in Figure 12 for various features observed on the solar photosphere. We found that for small amounts $([-0.03,0.03] \AA)$ of transmission profiles' displacement the variation of the brightness correction is negligible for features not affected by Doppler shifts (i.e., faculae and quiet sun). For line profiles shifted with respect to the quiet-Sun line center, a shift of filter center in the same direction as the line Doppler shift causes a reduction of the amount of correction, whereas a displacement in the opposite direction causes an increase. In addition, we found that broadening of the passband causes a decrease of the amount of correction when the width of the line profiles is larger than that of the passband. For transmission line profiles wider than the profile of the solar feature under study, the contrast is overestimated and the amount of deviation of the correction coefficient from unity increases with the increase of the filter degradation.

Our results are qualitatively in agreement with those presented by Mathew et al. (2007), who applied MDI procedure to synthetic line profiles obtained from Kurucz (1991) atmosphere models. Nevertheless, a comparison of results shows that they obtained brightness correction larger than the ones we have presented. These discrepancies must be ascribed to the differences in model atmospheres employed, assumptions for atomic parameters and micro and macro velocity values. Due to the arbitrariness of the choice of these quantities inherent to one-dimensional models, these analyses should be repeated by a direct comparison of MDI data with observations in the Ni I continuum properly compensated for scattered light, or with the employment of magneto-convection simulations encompassing a larger variety of sunspots.

Results presented in this study show that photometric measurements obtained with MDI data should be revised by taking into account the brightness correction factors presented. Due to the dependence of this factor on observational conditions, 
continuum images should be compensated for spatial scattered light before any correction is applied. Since MDI point-spread function varies with time, this result contributes to question studies based on the temporal variation of photometric properties of solar features derived from MDI data analyses.

Finally, our study proofs forward modeling as a powerful tool also for instrumental calibration. In particular, results presented here are of interest for the interpretation of data acquired with the Helioseismic and Magnetic Imager onboard the Solar Dynamic Observatory.

We thank Richard Wachter and Kevin Reardon for providing us with one of the IBIS data sets. This study was supported by the Istituto Nazionale di Astrofisica (PRIN-INAF-07), the Ministero degli Affari Esteri (Bando 2007 Rapporti bilaterali Italia-USA), and the Agenzia Spaziale Italiana (ASI/ESS/I/915/01510710). The NSO is operated by the Association of Universities for Research in Astronomy, Inc. (AURA), for the National Science Foundation. IBIS has been built by INAF/Osservatorio Astrofisico di Arcetri with contributions from the Universities of Firenze and Roma "Tor Vergata," the National Solar Observatory, and the Italian Ministries of Research (MUR) and Foreign Affairs (MAE).

\section{REFERENCES}

Berger, T., \& Lites, B. 2003, Sol. Phys., 213, 213

Bruls, J. H. M. J. 1993, A\&A, 269, 509

Briand, C., Mattig, W., Ceppatelli, G., \& Mainella, G. 2006, Sol. Phys., 234, 187

Cabrera Solana, D., Bellot Rubio, L. R., Beck, C., \& del Toro Iniesta, J. C. 2007, A\&A, 475, 1067

Cauzzi, G., Asensio Ramos, A., Reardon, K. P., \& Janssen, K. 2006, in ASP Conf. Ser. 354, Solar MHD Theory and Observations: A High Spatial Resolution Perspective, ed. J. Leibacher, R. F. Stein, \& H. Uitenbroek (San Francisco, CA: ASP), 26

Cauzzi, G., et al. 2008, A\&A, 480, 515

Cavallini, F. 2006, A\&A, 128, 589

Criscuoli, S., \& Ermolli, I. 2008, A\&A, 484, 591

Demidov, M. L., \& Balthasar, H. 2009, Sol. Phys., 260, 261

Fontenla, J. M., Avrett, E. H., \& Loeser, R. 1993, ApJ, 406, 319

Judge, P. G., Tritschler, A., Uitenbroek, H., Reardon, K., Cauzzi, G., \& de Wijn, A. 2010, ApJ, 710, 1486
Krivova, N. A., Solanki, S. K., Fligge, M., \& Unruh, Y. C 2003, A\&A, 399, L1

Kurucz, R. L. 1991, in Stellar Atmospheres: Beyond Classical Models, ed. L. Crivellari, I. Hubeny, \& D. G. Hummer (NATI ASI Series; Dordrecht: Kluwer), 440

Kurucz, R. L., Furenlid, I., Brault, J., \& Testerman, L. 1984, Solar Flux Atlas from 296 to $1300 \mathrm{~nm}$, National Solar Observatory Atlas (Sunspot, NM: National Solar Observatory)

Landi degli Innocenti, E., \& Landolfi, M. 2004, Polarization in Spectral Lines (Astrophysics and Space Science Library; Dordrecht: Kluwer)

Maltby, P., Avrett, E. H., Carlsson, M., Kjeldseth-Moe, O., Kurucz, R. L., \& Loeser, R. 1986, ApJ, 306, 284

Mathew, S. K., Martínez Pillet, V., Solanki, S. K., \& Krivova, N. A. 2007, A\&A, 465,291

Meunier, N., Roudier, T., \& Rieutord, M. 2008, A\&A, 488, 1109

Meunier, N., Roudier, T., \& Tkaczuck, R. 2007, A\&A, 466, 1123

Ortiz, A., Domingo, V., \& Sanahuja, B. 2006, A\&A, 452, 311

Ortiz, A., Solanki, S. K., Domingo, V., Fligge, M., \& Sanahuja, B. 2002, A\&A, 388, 1036

Rajaguru, S. P., Sankarasubramanian, K., Wachter, R., \& Scherrer, P. H. 2007, ApJ, 654, L175

Reardon, K. P., \& Cavallini, F. 2008, A\&A, 481, 897

Rees, D. E., \& Semel, M. D. 1979, A\&A, 74, 1R

Scharmer, G. B., Löfdahl, M. G., van Werkhoven, T. I. M., \& de la Cruz Rodriguez, J. 2010, A\&A, 521, A68

Scherrer, P. H., et al. 1995, Sol. Phys., 162, 129

Socas-Navarro, H., Martínez Pillet, V., Sobotka, M., \& Vázquez, M. 2004, ApJ, 614,448

Stein, R. F., \& Nordlund, A. 1998, ApJ, 499, 914

Stix, M. 2004, The Sun: An Introduction (Astronomy and Astrophysics Library; Berlin: Springer)

Tran, T., Bertello, L., Ulrich, R. K., \& Evans, S. 2005, ApJS, 156, 295

Uitenbroek, H. 2002, ApJ, 565, 1312

Uitenbroek, H. 2003, ApJ, 592, 1225

Ulrich, R. K., Bertello, L., Boyden, J. E., \& Webster, L. 2009, Sol. Phys., 255, 53

van Noort, M., Rouppe van der Voort, L., \& Löfdahl, M. 2005, Sol. Phys., 228, 191

Vitas, N., et al. 2010, A\&A, submitted

Viticchié, B., Del Moro, D., Berrilli, F., Bellot Rubio, L., \& Tritschler, A. 2009, ApJ, 700, L745

Wachter, R. 2008, Sol. Phys., 251, 491

Wachter, R., Schou, J., \& Sankarasubramanian, K. 2006, ApJ, 648, 1256

Wang, D., Zhang, M., Li, H., \& Zhang, H. Q. 2009, Sol. Phys., 260, 233

Wenzler, T., Solanki, S. K., \& Krivova, N. A. 2009, Geophys. Res. Lett., 36, L11102

Wenzler, T., Solanki, S. K., Krivova, N. A., \& Fröhlich, C. 2006, A\&A, 460, 583

Zhendong, G. G., Zhao, Y., \& Liu, Y. 2010, Res. Astron. Astrophys., 10, 1041 